\title{
Wind Power Short Term Forecasting based on Back Propagation Neural Network
}

\author{
Shenghui Wang ${ }^{1}$, Xiaonan Liu ${ }^{1}$, Yuexin Jin $^{1}$ and Keding $\mathrm{Qu}^{2}$ \\ ${ }^{1}$ Shenyang Institute of Engineering, Shenyang 110136, China; \\ ${ }^{2}$ NorthEast China Grid Company Limited, Shenyang 110136, China \\ * Corresponding author: liuxn0102@163.com
}

\begin{abstract}
This paper analyze and summarize the current situation as well as methods of forecasting wind power from home and aboard based on wind power development of China. Due to the BP neural network can approximate any nonlinear mapping with any arbitrary precision and its generalization ability is strong. This paper used BP neural network for power prediction, set up a model with numerical weather prediction data and wind power of a wind farm in Inner Mongolia Autonomous Region. Then used MATLAB to simulate and verify the feasibility of this prediction model the precision meet the requirements. In the last of this paper, the author development and design a simple system of wind power forecasting by using visual basic. The system has made the forecasting process to be simple and convenient, and also made easy to operation for the dispatcher.
\end{abstract}

Keywords: Wind power, Wind power forecasting, BPNN, Forecasting system

\section{Introduction}

With the rapid consumption of world energy and the deterioration of ecological environment, wind power as the major renewable energy has achieved a rapid development. And its development also attracted all countries attention. Chinese wind energy has a large reserve and wide distribution. The wind energy reserve in land has got about $2.53 \times 10^{8} \mathrm{KW}$. In the past decades, the global wind power cumulative installed capacity has eight-fold increase. Chinese wind power cumulative installed increase has increased 158 times and ranked first in the world.

According to the statistics that the Global Wind Energy Council (GWEC) has issued, the global cumulative installed capacity of wind power reached $3.18 \times 108 \mathrm{KW}$ at the end of 2013. Ranking the top five countries are China, America, Germany, Spain, India. Ranking the top five countries of the newly installed capacity are China, Germany, Britain, India, Canada. In 2013 year, the new installed capacity of China reached $1.609 \times 107$ million $\mathrm{KW}$ and the cumulative installed capacity reached $9.174 \times 10^{7} \mathrm{KW}$. The new grid connected capacity of wind power reached $1.449 \times 10^{7} \mathrm{KW}$, the cumulative grid connected capacity of $7.716 \times 10^{7} \mathrm{KW}$ [1]. Table 1 shows the situation of global accumulated and newly increased wind power capacity in 2013 of the first five countries.

Table 1. The Situation of Global Accumulated and Newly Increased Wind Power Capacity in $\mathbf{2 0 1 3}$ of the First Five Countries

\begin{tabular}{|c|c|c|c|c|c|}
\hline \multicolumn{3}{|c|}{ Accumulated wind power capacity in2013 } & \multicolumn{3}{c|}{ Newly increased wind power capacity in 2013} \\
\hline Ranking & Country & Capacity $\left(1 \times 10^{4} \mathrm{KW}\right)$ & Ranking & Country & Capacity $\left(1 \times 10^{4} \mathrm{KW}\right)$ \\
\hline 1 & China & 1609 & 1 & China & 9174 \\
\hline 2 & Germany & 324 & 2 & America & 6109 \\
\hline 3 & U.K & 188 & 3 & Germany & 3425 \\
\hline 4 & India & 173 & 4 & Span & 2296 \\
\hline 5 & Canada & 160 & 5 & India & 2015 \\
\hline
\end{tabular}


The wind power has a strong randomness and volatility. The unit capacity of wind turbine and the scale of grid wind farms has continuous expansion, so the wind power penetration is also increasing year by year. The attendant problems of wind power have become increasingly prominent. Therefore, the accurate forecasting of wind power is necessary to ensure the power system to be safe, reliable, economical and stable. It is also a problem that to be overcome urgently at this stage of China.

The wind power forecasting research abroad started very early, there are many mature commercial software has applied in power generation scheme and electric power market transaction, such as Prediktor of Denmark Risoe laboratory, WPPT and Zephyr of Technical University of Denmark and WPMS and Previento of Germany their wind power short-term forecast error can reach 10\%-15\%[2].Chinese wind power prediction started later than foreign. China has distinct climatic condition and geographic factors, we need to research and development our own wind power forecasting system. The first wind power forecasting system is developed by Chinese Electric Power Research Institute, named WPFS Ver1.0.It has been put into trial operation since 2008, its forecasting error reaches less than 20\%.The accuracy is in the domestic leading level, reached the level of similar foreign products[3]. Another forecasting system of wind power named WPFS was developed by North China Electric Power University, its rootmean-square error of Ultra short term forecasting has reached $10 \%$ within $6 \mathrm{~h}$. This system has been put into operation. In Longyuanchuanjing wind farms and Bayin wind farm of China Guodian Corporation [4]. In 2014,"VeStore-WPFS centralized wind power forecasting system V1.0" of Beijing Huadian Tianren Power Control Technology Co.,Ltd won the computer software copyright registration certificate which issued by the National Copyright Administration of the People's Republic of China.

According to the different forecasting time scale, Wind power prediction methods can be divided into ultra short term forecast, short-term prediction and long term prediction [5].According to the different forecasting models it can be divided into statistical model and physical model. According to different types of input data it can be divided into forecasting model based on historical data and forecasting model based on numerical weather prediction.

\section{Artificial Neural Network Model}

The ANN system has appeared since 1940s. Its principle is to imitate the structure and function of human brain. Large numbers of memory elements and topological elements are connecting to form the ANN model. The ANN has strong generalization ability and it is very effective to deal with nonlinear problems. Especially suitable for forecasting the intermittent and fluctuations wind power [6]. The ANN can approximate any nonlinear mapping with any degree of accuracy. The most mature applied in wind power forecasting is artificial neural network(ANN) and 80\% 90\% of the neural network model is Back Propagation neural network(BPNN) or its transformation[6].BPNN model algorithm of learning and training includes tow process, forward propagation process of input data and back propagation of output error. The essence of the algorithm is to adjust the weights and thresholds. The weights and thresholds of BPNN decrease along the fastest descent direction of performance function's gradient, it is the direction of the negative gradient [7].

\subsection{The Algorithm of BPNN}

Figure 1 is the structure of multi-input and single-output BPNN. 


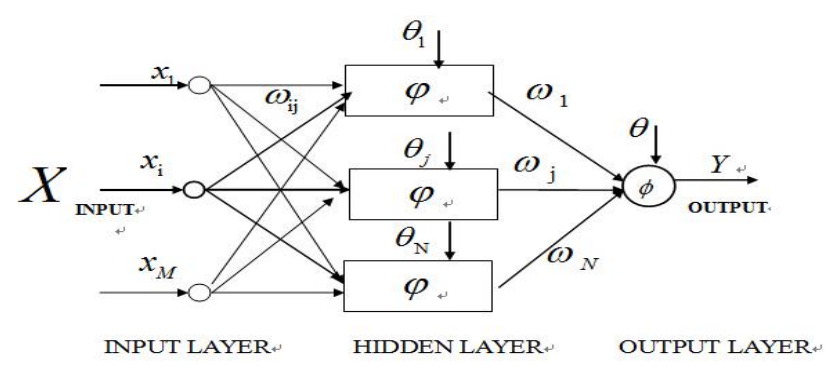

Figure 1. Structure of BPNN

$\alpha_{s}$ is the input of number $\mathrm{i}$ node in input layer $i=1,2, \ldots \ldots, M . \omega_{i j}$ is the weight between number $\mathrm{i}$ node in input layer and number $\mathrm{j}$ node in hidden layer.$\theta_{j}$ Is the threshold of number $\mathrm{j}$ node in input layer? $\varphi$ Is the transfer function in hidden layer? $\omega_{j}$ is the weight between number $\mathrm{i}$ node in hidden layer and the node in output layer, $j=1,2, \ldots \ldots, N \cdot \theta \cdot \theta_{j}$ Is the threshold of the node in output layer? $\phi$ is the transfer function in output layer. $Y$ is the output.

2.1.1. The Forward Propagation Process of Input: net $_{j}$ is the input of number $\mathrm{j}$ node in hidden layer.

$$
\text { net }_{j}=\sum_{i=1}^{M} \omega_{i j} x_{i}+\theta_{j}
$$

$o_{j}$ is the output of number $\mathrm{j}$ node in hidden layer.

$$
O_{j}=\varphi\left(\text { net }_{j}\right)=\varphi\left(\sum_{i=1}^{M} \omega_{i j}+\theta_{j}\right)
$$

net is the input of output node.

$$
\begin{aligned}
& n e t=\sum_{j=1}^{N} \omega_{j} O_{j}+\theta \\
& =\sum_{j=1}^{N} \omega_{j} \varphi\left(\sum_{i=1}^{M} \omega_{i j}+\theta_{j}\right)+\theta
\end{aligned}
$$

$\mathrm{Y}$ is the output of output node.

$$
Y=\phi(n e t)=\phi\left(\sum_{j=1}^{N} \omega_{j} \varphi\left(\sum_{i=1}^{N} \omega_{i j}+\theta_{j}\right)+\theta\right)
$$

2.1.2. The Back Propagation Process of Error: First of all, calculate the output node's error in output layer, the error in hidden layer and the error in input layer consequence. Then adjust the weights thresholds of each layer according to the error gradient descent method, so that the final output of the adjusted network can meet its expected value.

In the BPNN model, the sum number of sample date is $\mathrm{K}$, the desired output of each sample is y and the actual output of each sample is Y. So we define the error function as the sum of squares of the difference between $y$ and $Y$.

The error function of number $\mathrm{k}$ sample is

$$
E_{k}=\frac{1}{2}(y-Y)^{2}
$$

The total error function of total training samples is 


$$
E=\frac{1}{2} \sum_{k=1}^{K}\left(y_{k}-Y_{K}\right)^{2}
$$

Revise the weights and thresholds based on the error gradient descent method, ${ }^{\Delta \omega_{j}}$ is the weight's revised quantity of output layer, $\Delta \theta$ is the threshold's revised quantity of output layer, $\Delta \omega_{i j}$ is the weight's revised quantity of hidden layer, ${ }^{\prime}{ }_{j}$ is the threshold's revised quantity of hidden layer.

$$
\begin{array}{r}
\Delta \omega_{j}=-\eta \frac{\partial E}{\partial \omega_{j}}=\eta \sum_{k=1}^{K}\left(y_{k}-Y_{k}\right) \phi^{\prime}(\text { net }) O_{j} \\
\Delta \theta=-\eta \frac{\partial E}{\partial \theta}=\eta \sum_{k=1}^{K}\left(y_{k}-Y_{k}\right) \theta^{\prime}(\text { net }) \\
\Delta \omega_{i j}=-\eta \frac{\partial E}{\partial \omega_{i j}}=\eta \sum_{k=1}^{K}\left(y_{k}-Y_{k}\right) \phi^{\prime}(\text { net }) \omega_{j} \varphi^{\prime}\left(\text { net }_{j}\right) x_{i} \\
\Delta \theta_{j}=-\eta \frac{\partial E}{\partial \theta_{j}}=\eta \sum_{k=1}^{K}\left(y_{k}-Y_{k}\right) \phi^{\prime}(\text { net }) \omega_{j} \varphi^{\prime}\left(\text { net }_{j}\right)
\end{array}
$$

The new weights and thresholds of each layer are

$$
\begin{aligned}
& \omega_{j}=\omega_{j}+\Delta \omega_{j} \\
& \theta=\theta+\Delta \theta \\
& \omega_{i j}=\omega_{i j}+\Delta \omega_{i j} \\
& \theta_{j}=\theta_{j}+\Delta \theta_{j}
\end{aligned}
$$

\subsection{The Design of BPNN}

To establish a certain BPNN, we should determine the number of network layer and the number of nodes in each layer at first, then determine the transfer function and related parameters of each layer.

In general, with the increase of the layer number of the BPNN, the back propagation error of input layer will become less reliable. And also make it harder to get the ideal BPNN model. So the BPNN layer number generally select three. The number of the input layer is equal to the actual input parameters of involved problem, the number of output layer is equal to the number of the desired output parameters. In addition, there is no certain theory about the selection of BPNN's hidden layer's node number, mostly we determine it based on experience or trial. Many scholars believe that the node number of the hidden layer is equal to the node number of the input layer double times plus one [9]. The flow chart of establish a BPNN shows in Figure 2.

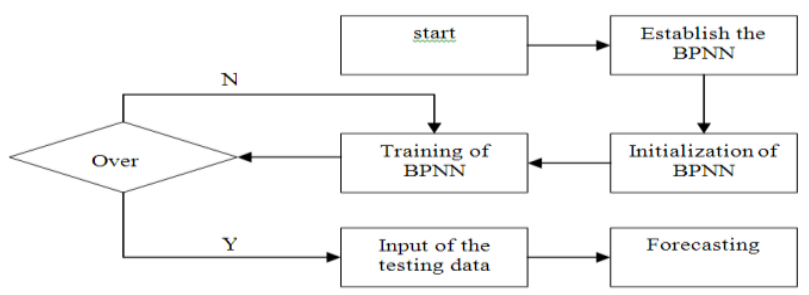

Figure 2. The Flow Chart Of Establish Neural Network 


\section{Reality of BPNN Models}

This paper establish the BPNN model to forecasting day-ahead wind power based on the numerical weather prediction data supplied by Shennengyihe wind farm of Shenzhen Energy North Holdings Co., Ltd in Inner Mongolia, China. The data includes numerical weather prediction data and real output of the wind farm between April 2 to 30.The data sample interval is $15 \mathrm{~min}$. There are 2784 groups of data in total, first 2688 data serve as training samples and the rest 96 samples serve as testing samples. This wind farm installs one hundred FD77B-1500 wind turbines produced by Dongfang turbine Co. Ltd. The unit capacity of the wind turbine is $1.5 \mathrm{MW}$ and the total installed capacity is $150 \mathrm{MW}$. Its theoretical output power characteristics curve under the standard condition $\left(\rho_{0}=1.225 \mathrm{~kg} / \mathrm{m}^{3}, T_{0}=288.15 \mathrm{~K}, p_{0}=101.33 \mathrm{KPa}\right.$ ) shows in figure 3.Its corresponding parameters shows in Table 2.

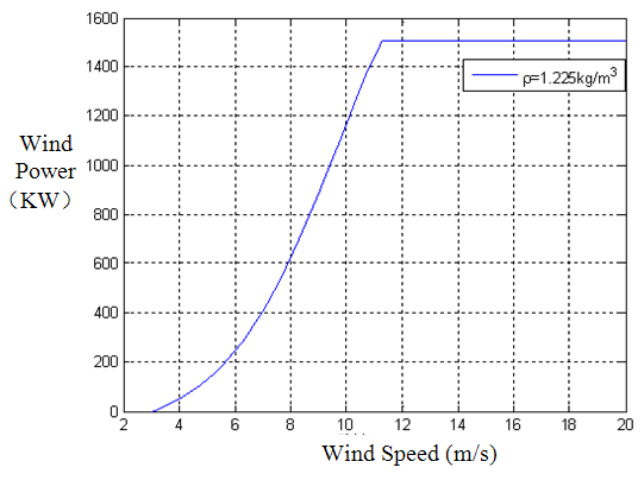

Figure 3. The Theoretical Output Power Characteristics Curve

Table 2. Parameters of FD77B-1500 Wind Turbine

\begin{tabular}{|c|c|}
\hline Type & FD77B-1500 \\
\hline Impeller number & 3 \\
\hline Rotor swept area & $4657 \mathrm{~m}^{2}$ \\
\hline Rated Power & $1.5 \mathrm{MW}$ \\
\hline Rated speed & $9.6 \sim 17.3 \pm 12 \% \mathrm{r} / \mathrm{min}$ \\
\hline Cut-in wind speed & $3 \mathrm{~m} / \mathrm{s}$ \\
\hline Cut-out wind speed & $20 \mathrm{~m} / \mathrm{s}$ \\
\hline Rated wind speed & $12.5 \mathrm{~m} / \mathrm{s}$ \\
\hline Rator diameter & $77 \mathrm{~m}$ \\
\hline Hub height & $65 \mathrm{~m}$ \\
\hline Operational temperature & $-40 \sim+40{ }^{\circ} \mathrm{C}$ \\
\hline
\end{tabular}

There is a problem need to pay more attention When establish the BPNN model to forecast the wind power. The excessive amplitude value of the input variable amplitude will make the neuron output saturation and influence the accuracy of the model. In order to improve the learning accuracy and efficiency of BPNN, all the sample data must be normalized before input to model. The network input and output sample value should limit into $[0,1]$ or $[-1,1]$. The mapminmax function of MATLAB can normalize the data into $[-1,1]$.Use this function to normalized the wind speed, temperature, pressure and actual power data. The wind direction normalizes with sine function and cosine function. After get the normalized forecasting value, we should reduce it to the original dimension. 
Normalize the data with formula (12), limit the data into $[-1,1]$.

$$
x_{i}=\frac{x_{i}-\frac{1}{2}\left(x_{\max }+x_{\min }\right)}{\frac{1}{2}\left(x_{\max }-x_{\min }\right)}
$$

Use formula (13) to reduce the normalization forecasting value to its original dimension.

$$
y_{i}=\frac{1}{2}\left(x_{\max }-x_{\min }\right) y_{i}^{\prime}+\frac{1}{2}\left(x_{\max }+x_{\min }\right)
$$

$x_{\max }$ And $x_{\min }$ is the maximum and minimum of the data, $x_{i}^{\prime}$ is the normalization value of input data and $y_{i}^{\prime}$ is the normalization value of output data.

In addition, the input data should be tested its accuracy before input the forecasting model. The data can not exceed its normal range. Table. 3 shows the range of each variable.

\section{Table 3. The Range of Each Variable}

\begin{tabular}{|c|c|}
\hline Inout data & Range \\
\hline Wind speed & $0 \mathrm{~m} / \mathrm{s} \sim 40 \mathrm{~m} / \mathrm{s}$ \\
\hline Wind direction & $0^{\circ} \sim 360^{\circ}$ \\
\hline Pressure & $94 \mathrm{kPa} \sim 106 \mathrm{kPa}$ \\
\hline Temperature & $-40^{\circ} \mathrm{C} \sim+50^{\circ} \mathrm{C}$ \\
\hline Relative Humidity & $1 \sim 100$ \\
\hline Wind power & $0 \mathrm{MW} \sim 150 \mathrm{MW}$ \\
\hline
\end{tabular}

The mainly input data in this forecasting model is the normalized numerical weather prediction data including wind speed, wind direction, temperature, Relative Humidity, and pressure. The wind speed is represented as sine and cosine value of the wind direction angle so the node number of input layer can be confirmed as 6 .According to the empirical formula, the node number of the hidden can be confirmed as 13 which equals the number of input nodes double times plus one. The node number of output layer can be confirmed as 1 . The model show in Figure 4.

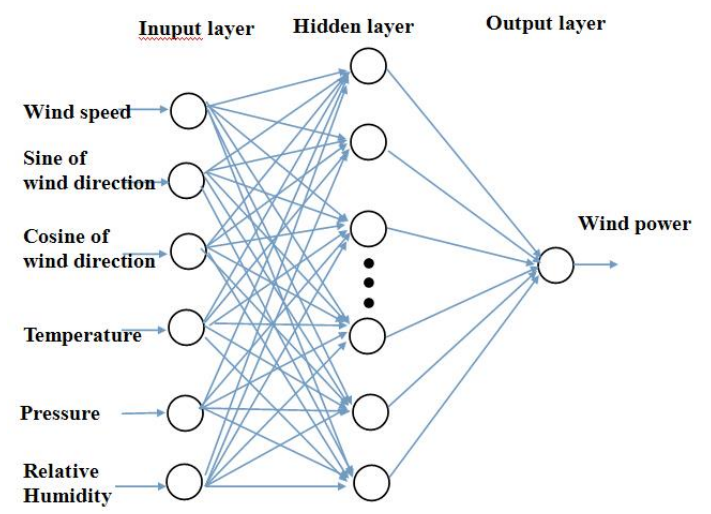

Figure 4. The Mode of BPNN

This paper constructs a BPNN model according to a practical problem. The transfer function of hidden layer and output layer adopts Tansig function, the training algorithm adopts trainlm function, the adjustment of the initial weights and thresholds adopts learngdm learning function and the error performance function adopts the MSE function. 
The maximum training time of this BPNN is 5000 , the training error is 0.005 , the learning rate is 0.05 .

Figure 5 is the comparison diagram between actual and prediction wind power. From this figure we can see the trend between the actual wind power and the outcome of the BPNN model is basically the same. Figure 6 is the error percentage of forecasting result.

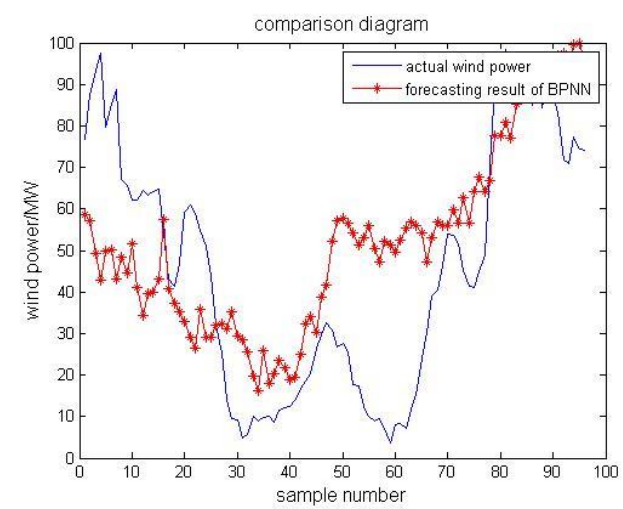

Figure 5. Comparison Diagram Between Actual and Prediction Wind Power

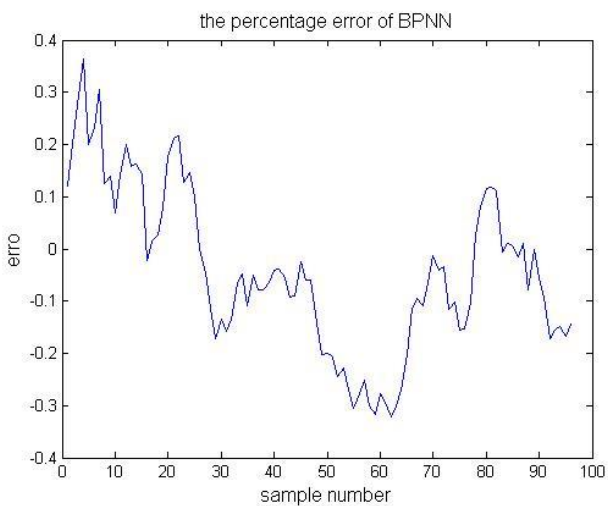

Figure 6. Comparison Diagram Between Actual and Prediction Wind Power

\section{The Development and Design of Forecasting System}

This paper uses visual basic to design a simple wind power forecasting system. Figure 7 is the Landing Interface When input user name as "liuxiaonan" and password as "1234567", the user can enter the wind power forecasting system. The system interface shows in Figure 8. Click on the menu bar button can operate it accordingly, including the data view and wind power forecasting.The results will be displayed in the interface.

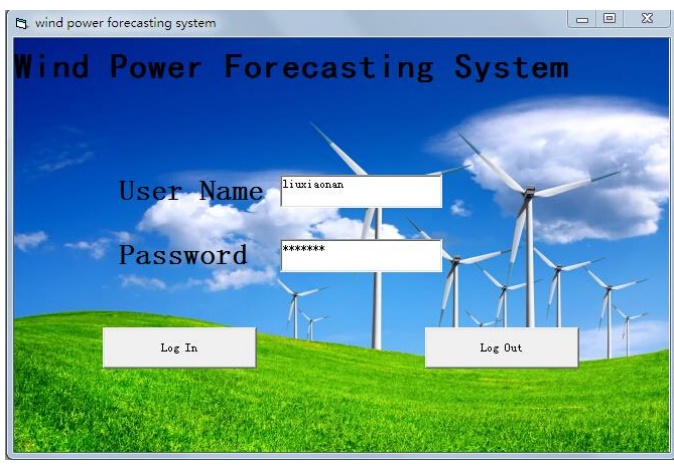

Figure 7. The Landing Interface 


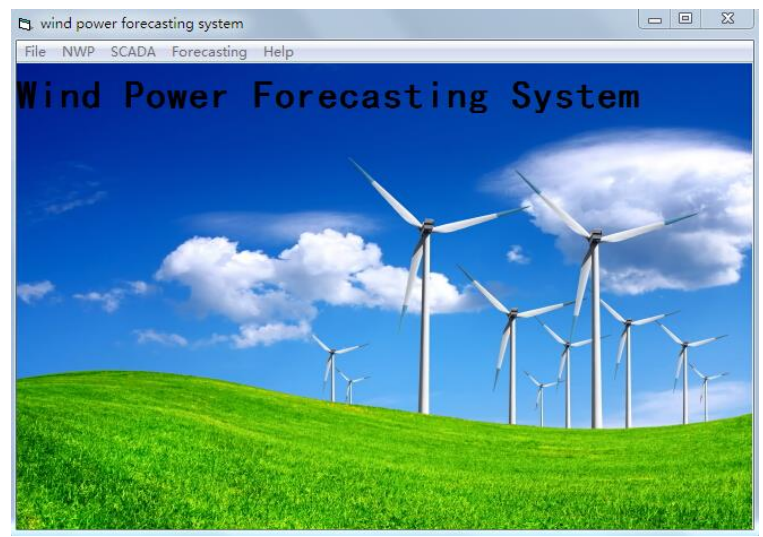

Figure 8. The Interface of the System

\section{Error Analysis of the Forecasting Results}

The evaluation index of wind power forecasting can be divided into error evaluation index and forecasting evaluation index. In this paper, we use the mean absolute error (MAE) and the root mean square error (RMSE) as the error evaluation index of forecasting results, the calculation formula show as follows.

$$
\begin{array}{r}
M A E=\frac{\sum_{t=1}^{T}\left|P_{t a}-P_{t f}\right|}{\operatorname{Cap} \times t} \\
R M S E=\sqrt{\frac{1 \sum_{t=1}^{T}\left(P_{t a}-P_{t f}\right)^{2}}{T \times C a p}}
\end{array}
$$

In the formula, $P_{t a}$ is the actual wind power of time $\mathrm{t}, P_{t f}$ is the forecasting wind power of time $\mathrm{t}, \mathrm{Cap}$ is the installed capacity, $\mathrm{T}$ is the total number of forecasting time.

The forecasting evaluation index include accuracy rate and qualified rate. It is mainly used to judge whether the forecasting result is meeting the grid requirements. According to relevant national regulations and standards, we can use the following formula to measure the accuracy rate and qualified rate of wind power forecasting.

$$
\begin{gathered}
r_{1}=\left\{\begin{array}{l}
\mid \sum_{t=1}^{\left.\frac{\sum_{t a}-P_{t p}}{C a p}\right)^{2}} \\
T
\end{array} \mid \times 100 \%\right. \\
r_{2}=\frac{1}{T} \sum_{t=1}^{T} B_{t} \times 100 \%
\end{gathered}
$$

In the formula $\left(1-\left|\frac{P_{t a}-P_{t f}}{C a p}\right|\right) \times 100 \% \geq 75 \%, B_{t}=1$, 


$$
\left(1-\left|\frac{P_{t a}-P_{t f}}{\operatorname{Cap}}\right|\right) \times 100 \%<75 \%, B_{t}=0 。
$$

The evaluation index of wind power forecasting in this paper shows in table4.

Table 4. The Evaluation Index

\begin{tabular}{|c|c|}
\hline Index name & Value \\
\hline MAE & $13.5 \%$ \\
\hline RMSE & $16.2 \%$ \\
\hline Accuracy rate & $83.8 \%$ \\
\hline Qualified rate & $85.4 \%$ \\
\hline
\end{tabular}

According to the Wind power forecasting management Interim Measures which issued by National Energy Administration of China[10], the maximum error of next-day forecasting curve provided by the wind power forecasting system should less than 25\%, the real-time forecasting error should less than $15 \%$, the root mean square error of all day should less than 20\%. The forecasting result of the BPNN model in this paper comply with the relevant requirements of the Interim Measures.And it also proves the practical feasibility of BPNN model to forecasting the wind power.

Under the situation about rapid change of wind speed, the forecasting error will increase and the generalization ability will decrease. The rapid fluctuation of wind speed can influence the forecasting performance of BPNN model. Not only the model structure and model parameter selection but also the accuracy of input data has an impact on the forecasting effects. So the input data should be tested its accuracy before input the forecasting model. The test include rejection of bad data and filling of missing data.

\section{Conclusion}

This paper presents the BPNN model and its algorithm. And also verifies the accuracy and practical value of BPNN forecasting model. The forecasting result of the BPNN model in this paper complies with the relevant requirements. Although artificial neural can approximate any nonlinear mapping with any arbitrary precision, but the traditional BP algorithm has a slow convergence rate and easily falls into local minimum, so many scholars have put forward more excellent forecasting method in wind power forecasting. With the continuous development of wind power forecasting technology, the forecasting method has changed from unicity to diversification. The combination forecasting method can make full use of each single method to reduce the error and improve the accuracy of forecasting results. This paper use Visual Basic to design and development a simple wind power forecasting system. The model has many places that need to be improved and perfected.

Wind power forecasting is a long-term and complicated work. From now on, we should work harder and continue to develop the forecast method which suitable for our national conditions and reality.

\section{Acknowledgements}

This work is supported by National Nature Science Foundation of China under Grant 61304069, 61372195, 61371200 the Nature Science \& Foundation of Liaoning Province under Grant 2013020124, the Key Technologies R\&D Program of Liaoning Province under Grant 2012201010. 


\section{References}

[1] “China Power News Net”, http://www.cpnn.com.cn/zdzgtt/201402/t20140226_657258.html

[2] "National Electric Power Dispatching and Communication Center of China State Grid Corp", Wind power forecasting system function norm, Beijing, China Electric Power Press, (2010).

[3] J. W. Fu and J. Ma, "Review of Wind Power Forecasting Methods", East China Electric Power, vol. 45, (2012).

[4] Q. Chen, "Review on Wind Power Prediction Technology Development", Shanxi Electric Power, vol. 172, (2012).

[5] M. Ahlstrom and R. M. Zavadil, "The Role of Wind Forecasting in Grid Operations and Reliability", Transmission and Distribution Conference and Exhibition, Asia and Pacific, vol. 1, (2005).

[6] G. F. Fan, W. S. Wang and C. Liu, "Short-term Wind Power Prediction System Based on Artificial Neural Network", Grid Technology, vol. 32, (2008), pp. 72.

[7] B. Zhou and L. Wen, "Application of BPNN in Wind Power Forecasting", Journal of Yibin University, vol. 12, (2012), pp. 30.

[8] J. L. Yang and X. J. Yang, "Artificial Neural Nets. Beijing”, Higher Education Press, (1992).

[9] Y. S. Xiao, W. Q. Wang and X. P. Huo, "Study on the Prediction of Wind Speed Time Series Based on Neural Network. Energy Conservation Technology", vol. 25, (2007), pp. 106.

[10] http://www.windpower.org.cn/news/lawsnews.jsp?id=176

\section{Authors}

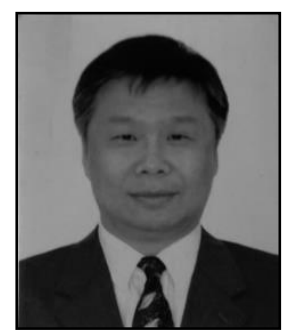

Shenghui Wang, received his PH.D degree from Shenyang University of Technology in 1999, and now is professor in Electrical Engineering department of Shenyang Institute of Engineering. His research interests include Application of PVSystems, and also electrical engineering control and automation.

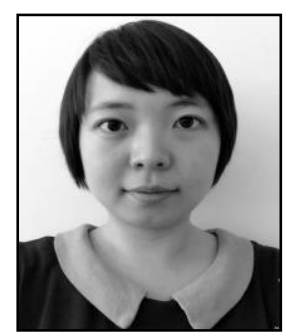

Xiaonan Liu, received her bachelor degree from Shanghai University of Electric Power in 2011, and now is doing further study in Shenyang Institute of Electrical Engineering. Her research interests include short term forecasting in wind power. 